Proyecciones Journal of Mathematics

Vol. 29, $\mathrm{N}^{o}$ 1, pp. 1-8, May 2010.

Universidad Católica del Norte

Antofagasta - Chile

\title{
SOME DIFFERENCE SEQUENCES DEFINED BY A SEQUENCE OF MODULUS FUNCTIONS
}

\author{
AHMAD H. A. BATAINEH \\ and \\ IBRAHIM M. A. SULAIMAN \\ AL AL-BAYT UNIVERSITY, JORDAN \\ Received : November 2009. Accepted : January 2010
}

\begin{abstract}
The idea of difference sequence spaces was introduced by Kizmaz [6], and this concept was generalized by Bektas and Colak [1]. In this paper, we define the sequence spaces $c_{0}\left(F, \Delta_{u}^{m} x\right)$ and $l_{\infty}\left(F, \Delta_{u}^{m} x\right)$, where $F=\left(f_{k}\right)$ is a sequence of modulus functions, and examine some inclusion relations and properties of these spaces.
\end{abstract}

Subjclass [2000] : 40A05, 40C05,46A45.

Keywords : Difference sequence spaces, A sequence of modulus functions. 


\section{Definitions and notations}

Let $w$ denote the set of all complex sequences $x=\left(x_{k}\right)$, and $l_{\infty}, c$, and $c_{0}$ be the linear spaces of bounded, convergent, and null sequences with complex terms, respectively, normed by $\|x\|=\sup _{k}\left|x_{k}\right|$, where $k \in \mathbf{N}$, the set of positive integers.

Kizmaz [6] defined the sequence spaces

$\mathrm{l}_{\infty}(\Delta)=\left\{x \in w: \Delta x \in l_{\infty}\right\}$,

$\mathrm{c}(\Delta)=\{x \in w: \Delta x \in c\}$, and

$\mathrm{c}_{0}(\Delta)=\left\{x \in w: \Delta x \in c_{0}\right\}$, where for any sequence $x=\left(x_{k}\right)$, the difference sequence $\Delta x$ is defined by

$$
\begin{aligned}
& \Delta x=\left(\Delta x_{k}\right)_{k=1}^{\infty}=\left(x_{k}-x_{k+1}\right)_{k=1}^{\infty}, \\
& \Delta^{0} x=\left(\Delta^{0} x_{k}\right)_{k=1}^{\infty}=\left(x_{k}\right)_{k=1}^{\infty} .
\end{aligned}
$$

Kizmaz [6] proved that these are Banach spaces with the norm $\|x\|=\mid$ $x_{1} \mid+\|x\|_{\infty}$. Also, he showed that $E \subset E(\Delta)$, where $E=\left\{l_{\infty}, c, c_{0}\right\}$, since there exists a sequence $x=\left(x_{k}\right)$ such that $x_{k}=k$, for each $k$, that is , $x=(1,2,3, \cdots)$ for which $\Delta x=(-1,-1,-1, \cdots)$, so that although $x$ is not convergent, but it is $\Delta$-convergent.

If $m$ is a nonnegative integer and $x=\left(x_{k}\right)$ is any sequence, then the difference sequences $\Delta x, \Delta^{2} x, \cdots, \Delta^{m} x$ are defined by

$\Delta x=\left(\Delta x_{k}\right)_{k=1}^{\infty}=\left(x_{k}-x_{k+1}\right)_{k=1}^{\infty}$,

$\Delta^{2} x=\Delta(\Delta x)=\Delta x_{k}-\Delta x_{k+1}$,

$\vdots$

$$
\Delta^{m} x=\Delta\left(\Delta^{m-1} x\right)=\Delta^{m-1} x_{k}-\Delta^{m-1} x_{k+1},
$$

so that

$\Delta^{m} x_{k}=\sum_{r=0}^{m}(-1)^{r}\left(\begin{array}{c}m \\ r\end{array}\right) x_{k+r}$.

Let $U$ be the set of all sequences $u=\left(u_{k}\right)$ such that $u_{k} \neq 0$ for each $k$. Then Gnanaseelan and Srivastava [4] defined and studied the sequence spaces

$\mathrm{E}(\mathrm{u}, \Delta)=\{x \in w: u \Delta x \in E\}$,

where $E=\left\{l_{\infty}, c, c_{0}\right\}$ and $u \Delta x=u_{k} x_{k}-u_{k+1} x_{k+1}$.

After then, Et. and Colak [2] defined the sequence spaces

$\mathrm{E}\left(\Delta^{m}\right)=\left\{x \in w: \Delta^{m} x \in E\right\}$,

where $E=\left\{l_{\infty}, c, c_{0}\right\}$.

They proved that these are Banach spaces with the norm $\|x\|=\sum_{i=1}^{m}$ | $x_{i} \mid+\left\|\Delta^{m} x\right\|_{\infty}$.

We recall that a modulus function $f$ is a function from $[0, \infty)$ to $[0, \infty)$ such that 
(i) $f(x)=0$ if and only if $x=0$,

(ii) $f(x+y) \leq f(x)+f(y)$ for all $x, y \geq 0$,

(iii) $f$ is increasing,

(iv) $f$ is continuous from the right at 0 .

Since $|f(x)-f(y)| \leq f(|x-y|)$, it follows from conditions (ii) and (iv) that $f$ is continuous everywhere on $[0, \infty)$.

A modulus function may be bounded or unbounded. For example, $f(t)=\frac{t}{t+1}$ is bounded but $f(t)=t^{p}(0<p \leq 1)$ is unbounded. Furthermore, we have $f(n x) \leq n f(x)$, from condition (ii), and so $f(x)=$ $f\left(n x \frac{1}{n}\right) \leq n f\left(\frac{x}{n}\right)$, hence $\frac{1}{n} f(x) \leq f\left(\frac{x}{n}\right)$, for all $n \in \mathbf{N}$.

Ruckle [13] used the concept of modulus function to define the sequence space

$\mathrm{L}(\mathrm{f})=\left\{\mathrm{x} \in w: \sum_{k=1}^{\infty} f\left(\left|x_{k}\right|\right)<\infty\right\}$.

If we take $f(x)=x^{p}$, then $L(f)$ reduces to the familiar space $l_{p}$ which is given by

$\mathrm{l}_{p}=\left\{x \in w: \sum_{k=1}^{\infty}\left|x_{k}\right|^{p}<\infty\right\}$.

In particular, if $f(x)=x$, then $L(f)=l_{1}$ which is given by

$\mathrm{l}_{1}=\left\{x \in w: \sum_{k=1}^{\infty}\left|x_{k}\right|<\infty\right\}$.

Several authors including Maddox ([8]-[11]), Ozturk and Bilgin [12], and some others studied some sequence spaces defined by a modulus function. Let $X$ be a sequence space. Then the sequence space $X(f)$ is defined by

$\mathrm{X}(\mathrm{f})=\left\{\mathrm{x} \in w: f\left(\left|x_{k}\right|\right) \in X\right\}$.

Kolk [7] gave an extension of $X(f)$ by considering a sequence of modulus functions $F=\left(f_{k}\right)$ and defined the space

$\mathrm{X}(\mathrm{F})=\left\{\mathrm{x} \in w: f_{k}\left(\left|x_{k}\right|\right) \in X\right\}$.

Gaur and Mursaleen [3] defined and studied the following sequence spaces

$l_{\infty}(F, \Delta)=\left\{x \in w: \Delta x \in l_{\infty}(F)\right\}$,

and

$\mathrm{c}_{0}(F, \Delta)=\left\{x \in w: \Delta x \in c_{0}(F)\right\}$.

For a nonnegative integer $m$, Bektas and Colak [1] extended the above mentioned spaces to

$1_{\infty}\left(F, \Delta^{m}\right)=\left\{x \in w: \Delta^{m} x \in l_{\infty}(F)\right\}$,

and

$\mathrm{c}_{0}\left(F, \Delta^{m}\right)=\left\{x \in w: \Delta^{m} x \in c_{0}(F)\right\}$.

We further give an extension of the spaces of Bektas and Colak [1] and define the sequence spaces 
$\mathrm{l}_{\infty}\left(F, \Delta_{u}^{m}\right)=\left\{x \in w: \Delta_{u}^{m} x \in l_{\infty}(F)\right\}$,

and

$\mathrm{c}_{0}\left(F, \Delta_{u}^{m}\right)=\left\{x \in w: \Delta_{u}^{m} x \in c_{0}(F)\right\}$,

where $u=\left(u_{k}\right)$ is any sequence such that $u_{k} \neq 0$ for each $k$, and

$\Delta_{u}^{0} x=u_{k} x_{k}$,

$\Delta_{u}^{1} x=u_{k} x_{k}-u_{k+1} x_{k+1}$,

$\Delta_{u}^{2} x=\Delta\left(\Delta_{u}^{1} x\right)$,

$\vdots$

$\Delta_{u}^{m} x=\Delta\left(\Delta_{u}^{m-1} x\right)$,

so that

$\Delta_{u}^{m} x=\Delta_{u_{k}}^{m} x_{k}=\sum_{r=0}^{m}(-1)^{r}\left(\begin{array}{c}m \\ r\end{array}\right) u_{k+r} x_{k+r}$.

If $u=e=(1,1,1, \cdots)$, then these spaces will give the spaces of Bektas and Colak [1] as special cases.

\section{Main results}

For a sequence $F=\left(f_{k}\right)$ of modulus functions, we will give the necessary and sufficient conditions for the inclusion between $X\left(\Delta_{u}^{m}\right)$ and $Y\left(F, \Delta_{u}^{m}\right)$, where $X, Y=l_{\infty}$ or $c_{0}$.

We need the following Lemmas ( see Kolk [7] ) :

Lemma 2.1. The condition $\sup _{k} f_{k}(t)<\infty, t>0$ holds if and only if there exists a point $t_{0}>0$ such that $\sup _{k} f_{k}\left(t_{0}\right)<\infty$.

Lemma 2.2. The condition $\inf _{k} f_{k}(t)>0, t>0$ holds if and only if there exists a point $t_{0}>0$ such that $\inf _{k} f_{k}\left(t_{0}\right)>0$.

Now, we prove the following theorems

Theorem 2.3. For a sequence $F=\left(f_{k}\right)$ of modulus functions, the following statements are equivalent :

(i) $l_{\infty}\left(\Delta_{u}^{m}\right) \subseteq l_{\infty}\left(F, \Delta_{u}^{m}\right)$

(ii) $c_{0}\left(\Delta_{u}^{m}\right) \subseteq l_{\infty}\left(F, \Delta_{u}^{m}\right)$

(iii) $\sup _{k} f_{k}(t)<\infty, t>0$ 
Proof. (i) implies (ii) is obvious

(ii) implies (iii) : Let $c_{0}\left(\Delta_{u}^{m}\right) \subseteq l_{\infty}\left(F, \Delta_{u}^{m}\right)$. Suppose that (iii) is not true. Then by Lemma 2.1, we see that $\sup _{k} f_{k}(t)=\infty$, for all $t>0$, and therefore there is a sequence $\left(k_{i}\right)$ of positive integers such that

$$
f_{k_{i}}\left(\frac{1}{i}\right)>i \text { for } i=1,2,3, \cdots
$$

Define $x=\left(x_{k}\right)$ as follows

$\mathrm{x}_{k}= \begin{cases}\frac{1}{i}, & k=k_{i} \\ 0, & \text { otherwise }\end{cases}$

Then $x \in c_{0}\left(\Delta_{u}^{m}\right)$, but by (2.1), $x \notin l_{\infty}\left(F, \Delta_{u}^{m}\right)$ which contradicts (ii). Hence (iii) must hold.

(iii) implies (i) : Let (iii) be satisfied and $\mathrm{x} \in l_{\infty}\left(\Delta_{u}^{m}\right)$. If we suppose that $x \notin l_{\infty}\left(F, \Delta_{u}^{m}\right)$, then $\sup _{k} f_{k}\left(\left|\Delta_{u}^{m} x_{k}\right|\right)=\infty$ for $\Delta_{u}^{m} x \in l_{\infty}$. Now take $t=\left|\Delta_{u}^{m} x\right|$, then $\sup _{k} f_{k}(t)=\infty$ which contradicts (iii). Hence $l_{\infty}\left(\Delta_{u}^{m}\right) \subseteq l_{\infty}\left(F, \Delta_{u}^{m}\right)$. This completes the proof of the theorem.

Theorem 2.4. For a sequence $F=\left(f_{k}\right)$ of modulus functions, the following statements are equivalent :

(i) $c_{0}\left(F, \Delta_{u}^{m}\right) \subseteq c_{0}\left(\Delta_{u}^{m}\right)$

(ii) $c_{0}\left(F, \Delta_{u}^{m}\right) \subseteq l_{\infty}\left(\Delta_{u}^{m}\right)$

(iii) $\inf _{k} f_{k}(t)>0,(t>0)$

Proof. (i) implies (ii) is obvious

(ii) implies (iii) : Let $c_{0}\left(F, \Delta_{u}^{m}\right) \subseteq l_{\infty}\left(\Delta_{u}^{m}\right)$. Suppose that (iii) does not hold. Then by Lemma 2.2 , we see that

$$
\inf _{k} f_{k}(t)=\infty,(t>0)
$$

and therefore there is a sequence $\left(k_{i}\right)$ of positive integers such that $\mathrm{f}_{k_{i}}\left(i^{2}\right)<\frac{1}{i}$ for $i=1,2,3, \cdots$.

Define $x=\left(x_{k}\right)$ as follows

$\mathrm{x}_{k}=\left\{\begin{array}{ll}i^{2}, & k=k_{i} \text { for } i=1,2,3, \cdots \\ 0, & \text { otherwise }\end{array}\right.$.

Then by (2.2), $x \in c_{0}\left(F, \Delta_{u}^{m}\right)$ but $x \notin l_{\infty}\left(\Delta_{u}^{m}\right)$ which contradicts (ii). Hence (iii) must hold.

(iii) implies (i) : Let (iii) be satisfied and $\mathrm{x} \in c_{0}\left(F, \Delta_{u}^{m}\right)$ that is $\lim _{k} f_{k}(\mid$ $\left.\Delta_{u}^{m} x_{k} \mid\right)=0$. Suppose that $x \notin c_{0}\left(\Delta_{u}^{m}\right)$, then for some number $\varepsilon_{0}>0$ and positive integer $k_{0}$, we have $\left|\Delta_{u}^{m} x_{k}\right| \varepsilon_{0}>0$ for $k \geq k_{0}$. Therefore $f_{k}\left(\varepsilon_{0}\right) \leq$ 
$f_{k}\left(\left|\Delta_{u}^{m} x_{k}\right|\right)$ for $k \geq k_{0}$ and hence $\lim _{k} f_{k}\left(\varepsilon_{0}\right)=0$ which contradicts (iii). Thus $c_{0}\left(F, \Delta_{u}^{m}\right) \subseteq c_{0}\left(\Delta_{u}^{m}\right)$ and this completes the proof of the theorem.

Theorem 2.5. The inclusion $l_{\infty}\left(F, \Delta_{u}^{m}\right) \subseteq c_{0}\left(\Delta_{u}^{m}\right)$ holds if and only if

$$
\lim _{k} f_{k}(t)=\infty \text { for } t>0
$$

Proof. Let $l_{\infty}\left(F, \Delta_{u}^{m}\right) \subseteq c_{0}\left(\Delta_{u}^{m}\right)$ and suppose that (2.3) does not hold . Then there is a number $t_{0}>0$ and a sequence $\left(k_{i}\right)$ of positive integers such that

$$
f_{k_{i}}\left(t_{0}\right) \leq L<\infty
$$

Define the sequence $x=\left(x_{k}\right)$ by

$\mathrm{x}_{k}= \begin{cases}t_{0}, & k=k_{i} \text { for } i=1,2,3, \cdots \\ 0, & \text { otherwise }\end{cases}$

Then $x \in l_{\infty}\left(F, \Delta_{u}^{m}\right)$ by (2.4) but $x \notin c_{0}\left(\Delta_{u}^{m}\right)$ so that (2.4) must hold if $l_{\infty}\left(F, \Delta_{u}^{m}\right) \subseteq c_{0}\left(\Delta_{u}^{m}\right)$.

Conversely, Suppose that $(2.3)$ is satisfied. If $x \in l_{\infty}\left(F, \Delta_{u}^{m}\right)$, then $f_{k}\left(\left|\Delta_{u}^{m} x_{k}\right|\right) \leq L<\infty$ for $k=1,2,3, \cdots$

Now suppose that $x \notin c_{0}\left(\Delta_{u}^{m}\right)$. Then for some $\varepsilon_{0}>0$ and positive integer $k_{0}$, we have $\left|\Delta_{u}^{m} x_{k}\right|>\varepsilon_{0}$ for $k \geq k_{0}$. Therefore $f_{k}\left(\varepsilon_{0}\right) \leq f_{k}(\mid$ $\left.\Delta_{u}^{m} x_{k} \mid\right) \leq L$ for $k \geq k_{0}$ which contradicts $(2.3)$. Hence $x \in c_{0}\left(\Delta_{u}^{m}\right)$.

Theorem 2.6. The inclusion $l_{\infty}\left(\Delta_{u}^{m}\right) \subseteq c_{0}\left(F, \Delta_{u}^{m}\right)$ holds if and only if

$$
\lim _{k} f_{k}(t)=0 \text { for } t>0
$$

Proof. Let $l_{\infty}\left(\Delta_{u}^{m}\right) \subseteq c_{0}\left(F, \Delta_{u}^{m}\right)$ and suppose that (2.5) does not hold . Then

$$
\lim _{k} f_{k}(t)=l \neq 0
$$

for some $t_{0}>0$.

Define the sequence $x=\left(x_{k}\right)$ by 
$\mathrm{x}_{k}=t_{0} \sum_{r=0}^{m}(-1)^{m}\left(\begin{array}{c}m+k-u-1 \\ k-u\end{array}\right)$ for $k=1,2,3, \cdots$.

Then $x \notin c_{0}\left(F, \Delta_{u}^{m}\right)$ by (2.6). Hence (2.5) must hold.

Conversely, Suppose that $(2.5)$ is satisfied. If $x \in l_{\infty}\left(\Delta_{u}^{m}\right)$, then | $\Delta_{u}^{m} x_{k} \mid \leq L<\infty$ for $k=1,2,3, \cdots$.

Therefore $f_{k}\left(\left|\Delta_{u}^{m} x_{k}\right|\right) \leq f_{k}(L)$ for $k=1,2,3, \cdots$ and $\lim _{k} f_{k}\left(\left|\Delta_{u}^{m} x_{k}\right|\right.$ )$\leq \lim _{k} f_{k}(L)=0$, by $(2.5)$. Hence $x \in c_{0}\left(F, \Delta_{u}^{m}\right)$.

\section{References}

[1] Bektas, Cigdem, A. and Colak, R., Generalized difference sequence spaces defined by a sequence of modulli, Soochow J. Math., 29(2), pp. 215-220, (2003).

[2] Et., M. and Colak, R., On some generalized difference sequence spaces, Soochow J. Math., 21, pp. 377-386, (1995).

[3] Gaur, A. K. and Mursaleen, Difference sequence spaces, Internat. J. Math. \& Math. Sci., 21(4), pp. 701-706, (1998).

[4] Gnanaseelan, C. and Srivastava, P. D., The $\alpha-, \beta-, \gamma-$ duals of some generalized difference sequence spaces, Indain J. Math., 38(2), pp. 111120, (1996).

[5] Kamthan, P. K. and Gupta, M., Sequence spaces and series, Lecture Notes in Pure and Applied Mathematics, vol. 65 , Marcel Dekker, New York, (1981).

[6] Kizmaz, H., On certain sequence spaces, Canad. Math. Bull. 24, pp. 169-176, (1981).

[7] Kolk, E., Inclusion theorems for some sequence spaces defined by a sequence of modulli, Acta Comment. Univ. Tartu, 970, pp. 65-72, (1994).

[8] Maddox, I. J., Some properties of paranormed sequence spaces, J. London Math. Soc. 1, pp. 316-322, (1969).

[9] Maddox, I. J. , Paranormed sequence spaces generated by infinite matrices, Proc. Camb. Phil. Soc., 64, pp. 431-435, (1969). 
[10] Maddox, I. J., Elements of Functional Analysis, 2nd Edition, Cambridge University Press, (1970).

[11] Maddox, I. J., Sequence spaces defined by a modulus, Math. Proc. Camb. Phil. Soc. 100, pp. 161-166, (1986).

[12] Ōztürk, E. and Bilgen, T., Strongly summable sequence spaces defined by a modulus, Indian J. Pure Appl. Math. 25(6), pp. 621-625, (1994).

[13] Ruckle, W. H., FK spaces in which the sequence of coordinate vectors is bounded, Canad. J. Math. 25, pp. 973-978, (1973).

\author{
Ahmad H. A. Bataineh \\ Department of Mathematics \\ Al al-Bayt University \\ P. O. Box 130095 Mafraq \\ Jordan \\ e-mail : ahabf2003@yahoo.ca \\ and
}

\title{
Ibrahim M. A. Sulaiman
}

Department of Mathematics

Al al-Bayt University

P. O. Box 130095 Mafraq

Jordan

e-mail : himo717@yahoo.com 